\title{
Musées et restes humains : Analyses comparées de cérémonies māori de rapatriement en sols québécois et français
}

\section{Natacha Gagné}

\section{(2) OpenEdition \\ Journals}

Édition électronique

URL : http://journals.openedition.org/jso/6984

DOI : $10.4000 /$ jso.6984

ISSN : $1760-7256$

Éditeur

Société des océanistes

Édition imprimée

Date de publication : 15 octobre 2013

Pagination : 77-88

ISBN : 978-2-85430-035-2

ISSN : 0300-953x

Référence électronique

Natacha Gagné, « Musées et restes humains : Analyses comparées de cérémonies māori de

rapatriement en sols québécois et français », Journal de la Société des Océanistes [En ligne], 136-137|

2013, mis en ligne le 20 mars 2014, consulté le 15 avril 2020. URL : http://journals.openedition.org/ jso/6984; DOI : https://doi.org/10.4000/jso.6984 


\title{
Musées et restes humains : analyses comparées de cérémonies māori de rapatriement en sols québécois et français ${ }^{1}$
}

par

\author{
Natacha GAGNÉ*
}

\section{RÉSUMÉ}

Le Museum of New Zealand Te Papa Tongarewa est responsable depuis 2003, au nom du gouvernement néo-zélandais, du rapatriement des kōiwi tangata (restes ancestraux) māori qui sont à l'étranger. Parmi les derniers rapatriements en liste, on retrouve des toi moko (têtes momifiées tatouées) ayant appartenus à des musées français et québécois. Les rapatriements donnent chaque fois lieu à des cérémonies dirigées par des Māori. Ce fut le cas au musée du quai Branly en janvier 2012 et au Musée des beaux-arts de Montréal en novembre 2012. Après une description de la cérémonie qui eut lieu à Montréal et une mise en exergue de ce qui la distinguait de celle qui se tint à Paris, cet article s'interrogera sur l'origine de ces différences. Elles seraient attribuables en partie aux contextes nationaux fort différents dans lesquels prenaient place les deux cérémonies.

Mots-CLÉs : musées, restes humains, Māori, France, Québec, décolonisation

Les vingt-et-un toi moko (têtes momifiées tatouées) restitués par la France à la NouvelleZélande en 2011 et $2012^{2}$ figurent parmi plus de 200 köiwi tangata (restes ancestraux) māori rapatriés par le Museum of New Zealand Te

\begin{abstract}
Since 2003, the Museum of New Zealand Te Papa Tongarewa is responsible, on behalf of the New Zealand government, of the repatriation of kōiwi tangata Māori (Mãori human remains) from overseas. The recent repatriations include toi moko (tattooed mummified heads) which were housed by French and Québécois museum. Each repatriation is comprised of a ceremony under Mãori leadership. It was the case at the Musée du quai Branly in January 2012 and at the Montreal Museum of Fine Arts in November 2012. After a description of the ceremony that took place in Montreal and the highlighting of what distinguish it from the ceremony that took place in Paris, this paper will seek to interrogate the origin of the differences. They might be explained in part by the very different national contexts in which both ceremonies took place.
\end{abstract}

KeYwords: Museum, Human remains, Māori, France, Quebec, Decolonization

Papa Tongarewa (Te Papa) en provenance de quatorze pays ${ }^{3}$. Ce musée possède en effet depuis 2003 la responsabilité des rapatriements des kōiwi tangata māori au nom du gouvernement néo-zélandais.

1. Cette recherche fut rendue possible grâce à une subvention ordinaire du Conseil de la recherche en sciences humaines du Canada (CRSH).

2. Pour des détails, voir Rostkowski (2010), Boulay (2012) et Gagné (2012).

3. Voir la section "International Rapatriations » du site internet du Te Papa à l'adresse suivante http://www.tepapa. govt.nz/AboutUs/Repatriation/Pages/InternationalRepatriations.aspx (site consulté le 15 janvier 2013). Pour une chronologie générale concernant les toi moko, voir Peltier et Mélandri (2012).

* Professeure, Département d'anthropologie, Université Laval, natacha.gagne@ant.ulaval.ca. 
Les tout derniers rapatriements en liste eurent lieu en Amérique du Nord (Canada et ÉtatsUnis), à l'occasion du déplacement d'une équipe $\mathrm{du}$ Te Papa dans le cadre de l'installation et de l'inauguration de l'exposition itinérante $E$ tù ake : Standing Strong qui sera à l'affiche au $\mathrm{Mu}$ sée de la civilisation de Québec (MCQ) jusqu'au 8 septembre 2013 sous le titre $E$ tù ake: Mãori debout. C'est d'ailleurs à l'occasion de la clôture de cette même exposition - alors intitulée Māori : leurs trésors ont une âme - au Musée du quai Branly (MQB) que se tint, en janvier 2012, la cérémonie de restitution de vingt toi moko dont j'ai rendu compte dans un numéro précédent de la présente revue (Gagné, 2012).

Dans les pages qui suivent, je m'intéresserai à la cérémonie officielle de remise d'un toi moko au Te Papa par le Musée des beaux-arts de Montréal (MBAM) le 21 novembre 2012 pour la comparer avec la cérémonie qui se tint au MQB au début de la même année. Je donnerai tout d'abord quelques éléments d'information sur le toi moko conservé à Montréal et la demande de rapatriement dont il fit l'objet. Je décrirai par la suite en détail le déroulement de la cérémonie de rapatriement et je ferai ressortir ce qu'elle eut en commun et en quoi elle se distingua de la cérémonie qui se tint à Paris. Dans une dernière partie, je donnerai quelques précisions sur les contextes québécois et canadiens plus larges dans lesquels s'est déroulée la cérémonie au мвАм, lesquelles fourniront des éclairages sur sa forme et son contenu particuliers.

\section{Le toi moko conservé au Musée des beaux- arts de Montréal}

Le MBAM ainsi que d'autres musées canadiens reçurent en 2008 une lettre en provenance du Te Papa demandant le rapatriement des köiwi tangata māori en leur possession. Ces demandes $s$ 'inscrivaient dans le cadre du programme Karanga Aotearoa ${ }^{4}$ chargé d'inviter les établisse- ments étrangers qui possèdent des köiwi tangata à les rapatrier.

Le toi moko qui fut restitué le 21 novembre dernier par le мВАм fut le cinquième et dernier à être rapatrié de ceux conservés dans les collections des musées canadiens. Les autres appartenaient au Musée canadien des civilisations (Gatineau, Québec) et au Musée royal de l'Ontario (Toronto, Ontario) et furent rapatriés en 2008, en même temps que trois fragments humains qui étaient conservés au Museum of Anthropology de l'University of British Columbia (Vancouver, Colombie-Britannique). Le toi moko du MBAm fut un don de F. Cleveland Morgan qui l'avait acquis en août 1949 des Berkeley Galleries de Londres. Il fut exposé en salle au мвам entre 1982 et 1984 et fut conservé par la suite dans les réserves du musée.

Nathalie Bondil, directrice et conservatrice en chef du MBAM, explique comment fut reçue la demande du Te Papa :

"Au cours des recherches que nous avons menées sur nos collections des cultures du monde pour réaliser l'an dernier le Musée réinventé, une réponse positive à la demande du Te Papa s'est imposée " (мвам, communiqué de presse, 21 novembre 2012)

La demande de rapatriement fut alors acheminée au Conseil d'administration du MBAM qui vota à l'unanimité en sa faveur (idem). Le MCQ aurait par la suite facilité le contact entre les deux musées en vue d'une entente sur le déroulement de la procédure de rapatriement. Sur les ondes de свс Radio, Hélène Bernier, directrice des expositions et des affaires internationales au MCQ, expliqua que dans les échanges entourant l'exposition $E$ tu ake, le musée de Québec avait été informé par le Te Papa de la présence du toi moko au musée montréalais et de leur demande de rapatriement. Combiner les deux événements sembla une excellente idée aux responsables du MBAM, selon Nathalie Bondil, s'exprimant à la même émission ("Cinq à Six ", CBC Radio, 27 novembre 2012) ${ }^{6}$.

4. Une traduction du nom de ce programme serait "Un appel de bienvenue de la Nouvelle-Zélande ». Le karanga est l'appel cérémoniel fait par une femme (habituellement âgée) pour appeler les visiteurs, mais également les esprits des ancêtres sur le marae lors d'un rituel de rencontre (Salmond 1975). Voici comment est expliqué ce choix de nom dans un rapport émanant du programme : "The karanga (beckoning call) is a spiritual dialogue, imbued with sacredness it reunites the living with their loved ones who have passed on under the mantel of their eponymous ancestry (Te-Hono-ki-Hawaiki) and within their homelands (Aotearoa, Te Waipounamu me Rekohu)" (http://www.tepapa.govt.nz/SiteCollectionDocuments/ AboutTePapa/Repatriation/TairawhitiHuiaRoheReport.pdf).

5. Communiqué de presse disponible sur internet : http://www.mbam.qc.ca/bibliotheque/media/Communique-ToiMoko-mbam.pdf (site consulté le 15 janvier 2013). Le Musée réinventé fut un projet de réaménagement complet du MBAM complété en 2011 "avec l'ouverture du nouveau pavillon d'art québécois et canadien Claire et Marc Bourgie, l'inauguration d'une nouvelle salle de concert, le dévoilement d'un pavillon entièrement dédié aux arts décoratifs et au design et le redéploiement de toutes ses collections d'art international " (MBAM, communiqué de presse, 26 septembre 2011, disponible à : http://www.mbam.qc.ca/documents/10367/f1f9d609-d52a-47cf-980c-289a4cfbff22 (site consulté le 15 janvier 2013).

6. Disponible sur internet: http://www.cbc.ca/cinqasix/cultural-institutions/2012/11/27/museums-our-vision-ischanging-towards-other-cultures/ (site consulté le 15 janvier 2013). СвC est le pendant anglophone de Radio-Canada, le radiodiffuseur public national du Canada. 


\section{Déroulement de la cérémonie au MBAM}

Le lendemain de l'inauguration de l'exposition E tù ake: Māori debout à Québec le 20 novembre 2012, une partie de la délégation néo-zélandaise se déplaça à Montréal, accompagnée de Michel Côté, directeur général du MCQ, et d'autres représentants du MCQ, en vue de la cérémonie officielle au cours de laquelle le MBAM remit le toi moko à la délégation néo-zélandaise. Faisait également partie de la délégation M. Winton Holmes, hautcommissaire de la Nouvelle-Zélande à Ottawa, qui s'était également adressé au public lors de la cérémonie de la veille au MCQ.

À leur arrivée au musée, les invités à la cérémonie, les membres de la délégation néo-zélandaise et les membres de la presse se virent offrir du café, des boissons ainsi que des pâtisseries dans le hall d'entrée du pavillon Michal et Renata Hornstein du mвам. À l'approche de l'heure du début de la cérémonie, les invités et la presse soit une trentaine de personnes - furent conviés à se diriger vers l'endroit où devait se tenir la cérémonie, au cœur du musée, au bas des escaliers menant vers les salles. Le lieu était interdit d'accès au public. On n’y retrouvait aucun élément de décor ajouté pour l'occasion. Le caractère dépouillé du lieu contrastait avec la décoration de la scène du théâtre Claude Lévi-Strauss en janvier 2012 au MQB où l'on pouvait voir six portraits grand format d'ancêtres māori, des éléments végétaux, une projection d'un paysage de la côte néo-zélandaise, auxquels s'ajoutaient des effets d'éclairage donnant à l'ensemble un aspect théâtralisé. A Montréal, malgré l'absence de décor, le lieu était empreint d'un caractère solennel puisqu'au milieu de la salle, face aux sièges réservés au public et entre les deux rangées de sièges réservés, d'un côté, à la délégation néo-zélandaise - les manuhiri ou invités - et, de l'autre, aux représentants du musée et au représentant du gouvernement du Québec - les hunga käinga ou gens du lieu, hôtes -, reposait sur une table le toi moko recouvert d'une voilette noire semi-transparente. Cette présence incitait au silence et au recueillement. À notre entrée dans la salle, un responsable nous avisa qu'il était interdit de prendre des photos ou de filmer avant que le toi moko ne soit déposé et soigneusement emballé dans la boîte de transport à l'intérieur de laquelle il ferait son voyage de retour vers la Nouvelle-Zélande.

Avant le début de la cérémonie à proprement parler, alors que tout le monde avait pris place et que la délégation māori attendait à l'extérieur de la salle, Michelle Hippolite, kaihautū (leader māori) dans l'équipe de direction du Te Papa, prit la parole pour expliquer aux personnes présentes le déroulement général de la cérémonie, donnant des précisions sur chaque moment de la séquence rituelle et sa signification, afin que les gens comprennent ce qui allait se passer et d'éviter les surprises. Cette intervention marquait déjà un contraste important avec ce qui s'est passé lors de la cérémonie au MQB où aucune explication ne précéda l'entrée dans la salle de la délégation māori. Elle expliqua que les membres de la délégation allaient faire leur entrée au son des conques et que la procession allait se diriger vers le tipuna, l'ancêtre, le toi moko alors exposé en chapelle ardente, en faisant des incantations.

Ces explications rendaient explicite un des take (objet, motif) du rituel de rencontre ${ }^{7}$ : marquer le passage d'une personne de la vie à la mort. Il s'agissait donc d'un rituel de funérailles. L'explication donnée par Mme Hippolite quant à l'interdiction de prendre des photos du toi moko était tout à fait claire à cet effet :

"The reason why we ask you to respect no clicking while he is laying in state is because he is still an ancestor that is passed away and in our culture, when there are photos taken when somebody dies it is not of the person himself, but usually of the family that are morning in grief along with that person. At the moment, he is just here by himself while we are here to come and pick him up."

Michelle Hippolite identifia également le deuxième take (objet, motif) de la rencontre qui allait faire l'objet d'une attention particulière dans un second temps : l'entente de rapatriement du toi moko et sa signature. Les deux take correspondent d'ailleurs à deux moments distincts du rituel de rencontre qui se distinguent par leur atmosphère générale: dans une première partie, toute l'attention fut donnée à l'ancêtre décédé. La tristesse quant à sa mort fut exprimée par des larmes et le gémissement de la conque. Des chants et des prières furent exécutés afin de s'assurer de son retour en toute sécurité vers la terre de ses ancêtres, dans l'attente de la suite de la cérémonie de funérailles et de son repos final. Comme l'expliqua plus tard Michelle Hippolite pendant la période des mibimibi (salutations cérémonielles, échanges oratoires), peu d'informations étant connues sur cet ancêtre, il reposera pendant quelque temps encore dans un wāhi tapu (lieu, dépôt sacré) au musée Te Papa jusqu’à ce que ses origines tribales soient connues, qu il soit rendu à sa tribu et enterré dans le respect des coutumes.

Le ton de la rencontre changea progressivement et se fit plus joyeux au fur et à mesure du

7. Tel que déjà mentionné dans Gagné (2012 : 11-12), Salmond (1975 : 179) identifie deux grandes classes de take: ceux dont l'événement principal est lié aux étapes importantes de la vie (funérailles, $21^{\mathrm{e}}$ anniversaire de naissance, mariage, etc.) et qui se focalisent sur un ou des individus et ceux dont l'événement principal implique un groupe. Les deux classes de take furent présentes dans les cérémonies de rapatriement à Paris et à Montréal. 
déroulement du rituel de rencontre. Sans jamais que l'ancêtre n'y perde sa place centrale, la rencontre fut également l'occasion de réjouissances puisque le tipuna pourrait enfin rentrer chez lui. Comme l'expliqua Rahui Papa, le kaumatua (aîné) et expert culturel en charge de la cérémonie pendant les mibimibi:

"This ancestor has had a wonderful holiday in Montreal, an extended long holiday, but a holiday all the same. [...] This ancestor is able to return to the shores of New Zealand, to the mountains, to the forests, to the very land from which he once came. [...] Now we have the opportunity to take him home."

Mais il y avait également une autre raison de se réjouir : une nouvelle relation avait été nouée, un dialogue avait été établi. Un koha, un présent, sera d'ailleurs remis aux représentants du MBAM par les manuhiri (visiteurs) en signe de réciprocité et dans le but de pérenniser la relation. Voici ce que Rahui Papa en dit au moment de l'offrir :

"We also offer something to the Museum of fine arts as a token... a small token gesture of our affection, of our thanks because we have come together as one people."

Un peu plus tôt dans la cérémonie, il avait d'ailleurs manifesté au musée et à ses représentants toute sa reconnaissance de la part du Te Papa, du peuple māori et de toute la nation néozélandaise pour ce fantastique taonga (trésor) qu'ils pouvaient maintenant ramener chez eux :

"Kua rangatira mätou i tenei rā. We have become chiefs with your warm hospitality and your agreement to allow this ancestor to make his travel home."
Les rituels de rencontre de façon générale permettent de sceller les relations entre les groupes en présence (Metge, $1976: 234$, Tauroa et Tauroa, 1986 : 89-90). Comme le précise Rosenblatt (2011 : 418), sur un marae, le lieu traditionnel de rencontre des Māori où se tiennent habituellement les rituels, après la cérémonie d'accueil des visiteurs, ces derniers ne forment plus qu'un seul groupe avec leurs hôtes et cette unité est comprise comme une parenté temporaire, laquelle est mise en pratique et renforcée pendant toute la durée de la rencontre.

Comme je l'ai fait dans le cas de la cérémonie de rapatriement ayant eu lieu au MQB, j'en viens à présent aux détails des différents moments de la cérémonie de rapatriement qui se tint au MBAM, c'est-à-dire aux éléments ou unités composant la séquence rituelle. La cérémonie à proprement parler débuta après les explications de Michelle Hippolite et son départ de la salle pour rejoindre son groupe. On entendit alors retentir la conque qui servait, comme indiqué par Mme Hippolite, " to open the pathway", à dégager la voie de tout obstacle ou influence surnaturel pouvant être néfaste. Tout le monde dans l'assistance se leva alors, tel que l'avait demandé Mme Hippolite. Le kaumatua Rahui Papa était au-devant de la procession, suivi des femmes du groupe, puis des hommes. Alors que résonnait toujours la conque, une femme du groupe exécuta un karanga, un appel chanté à l'intention de l'ancêtre qui reposait en chapelle ardente. Dès le karanga terminé, Rahui Papa récita une incantation, alors que la délégation s'approchait du toi moko. L'émotion était palpable dans la salle et des larmes coulaient sur les joues de certains membres du groupe des

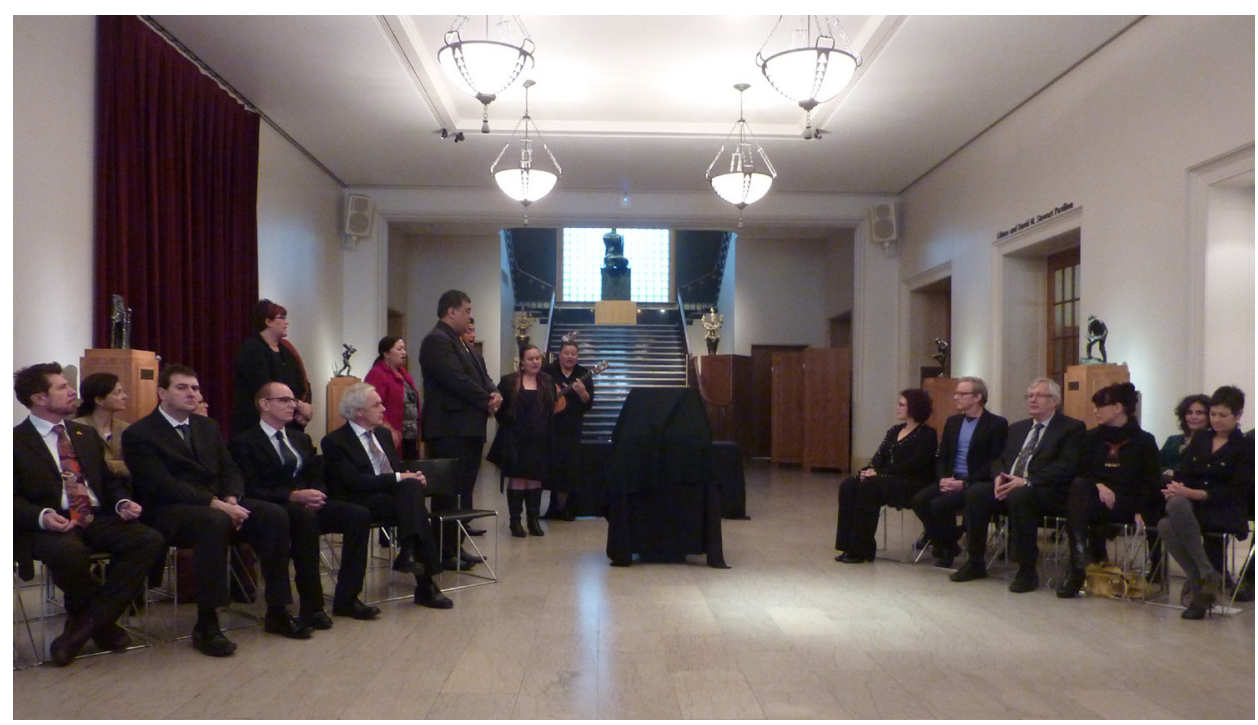

Рното 1. - Chant concluant la première partie de la cérémonie (21 novembre 2012, cliché Natacha Gagné). Les chants ont lieu après que le toi moko, au centre de la photo, a été déposé dans sa boîte de transport et recouvert d'un drap mortuaire. Debout, immédiatement derrière le groupe des visiteurs qui sont assis sur la gauche, Michelle Hippolite et Rahui Papa. 
manubiri. C'est d'ailleurs ce qui fera dire plus tard à la directrice du мвАм, Mme Bondil :

"We were all very moved by the ceremony. [...] listening to the songs... It was so moving, so sad at some point. People were even crying. [...] We could feel that it was the right decision. " ("Cinq à Six », СВC Radio, 27 novembre 2012)

Comme je l'ai fait remarquer à propos de la cérémonie au $\mathrm{MQB}$ en 2012, la symbolique du tangihanga (funérailles) rendait d'autant plus forte l'affirmation de leur autorité par les Māori, tant par l'intensité dramatique de la cérémonie que par la légitimité que fournit au groupe l'ancrage dans les traditions ancestrales.

La conque gémissait en accompagnement de l'incantation, imitant, me semble-t-il, les pleurs et les cris des femmes endeuillées appelées pani qu'on retrouve lors de tangihanga (funérailles) sur les marae. Des pani figuraient d'ailleurs dans la cérémonie $\mathrm{au} \mathrm{MQB}$ et passèrent toute la cérémonie agenouillées ou assises près des toi moko, au centre de la scène (voir photos dans Gagné, 2012). Le tangi (gémissements et pleurs), de façon générale, est un élément important des rituels de rencontre māori qui fournissent l'occasion de se remémorer les morts et d'exprimer son regret par rapport aux êtres aimés disparus.

Le groupe s'étant rapproché du toi moko, les membres non māori de la délégation prirent place sur les sièges leur étant réservés, alors que les Māori du groupe restèrent debout derrière leur kaumatua (aîné) qui se tenait tout près du toi moko. Ils chantèrent par la suite une waiata tangi, une chanson de deuil ou de lamentation, alors qu'une membre de l'équipe du MBAM, aidée par une membre de l'équipe du Te Papa, emballait et mettait avec grand soin le toi moko dans sa boîte de transport qui fut par la suite recouverte d'un tissu noir faisant office de drap mortuaire. Il est à noter que tous les chants furent exécutés sous la direction d'une des femmes du groupe. Sauf dans le cas de la waiata tangi exécutée a cappella, c'est aussi elle qui accompagna les chants à la guitare.

Rahui Papa s'adressa par la suite au tipuna (ancêtre), le conviant au voyage de retour vers la terre de ses ancêtres. Un chant suivit en conclusion de cette première partie de la cérémonie, laquelle visait surtout à répondre au premier take (objet, motif) (voir photo 1). Depuis le début de la cérémonie jusqu'à ce moment précis, tout se passa en langue māori. Chaque élément avait pourtant été expliqué en anglais auparavant par Michelle Hippolite et certaines précisions avaient également été données en français par une responsable du MBAM juste après son intervention.
Le deuxième temps de la cérémonie, davantage orienté par le deuxième take (objet, motif), s'ouvrit avec les mihimihi ${ }^{8}$ ou échanges oratoires entre le groupe hôte et les invités. La directrice du Musée des beaux-arts de Montréal, Nathalie Bondil, principale représentante du groupe hôte, prit la parole en premier, comme c'est l'usage. Dans une courte allocution prononcée en anglais et en français, Mme Bondil exprima sa joie et expliqua pourquoi le Conseil d'administration du musée s'était prononcé en faveur de la demande de rapatriement. Elle insista également sur les changements remarqués au cours des dernières années dans le milieu muséal quant aux traitements réservés aux restes humains. L'allocution de Mme Bondil fut immédiatement suivie par celle de Rahui Papa. Il parla surtout en anglais après quelques mots en māori, faisant rire l'assistance en disant qu'il avait oublié son français à Québec, contribuant ainsi à alléger l'atmosphère de la rencontre9. Michelle Hippolite prit la parole par la suite pendant un peu plus d'une dizaine de minutes, revenant entre autres sur l'histoire des toi moko depuis le $\mathrm{XIX}^{\mathrm{e}}$ siècle, sur les changements en muséologie et les difficultés de documenter certains restes humains, en particulier celui qui était au centre de cette cérémonie. Toujours du côté du groupe des manuhiri (invités), le hautcommissaire de la Nouvelle-Zélande dit par la suite quelques mots en māori et en français pour souligner l'importance de l'entente. Michel Côté du MCQ prit la parole le dernier en français, exprimant sa reconnaissance au peuple māori de mener le combat vers l'autodétermination, un peuple dont la culture, respectueuse du passé, est toujours vivante. Un chant vint par la suite marquer la fin des mibimibi.

Rahui Papa reprit la parole ensuite pour expliquer les deux prochains éléments de la séquence rituelle, soit la remise d'un koha (présent), que nous avons déjà évoquée plus haut, qui prit la forme d'une sculpture en bois, laquelle fut suivi du hongi (voir photo 2). Il expliqua que le hongi est :

" the pressing of the noses, it's the joining of the life force or the breath of life that [weaves] us all as one together because we believe that we don't come by ourselves, we believe we carry on our shoulders our ancestors from times immemorial and so that's just make this even more special today. So we want to share the hongi and the rūrū [se serrer la main] culture with our lovely friends from Montreal."

Comme c'est le cas habituellement sur un marae, le groupe des visiteurs se déplaça pour rencontrer et faire le hongi à chacun des membres du groupe des hôtes. Cet élément de la séquence

8. Dans des contextes plus formels - par exemple, sur un marae "traditionnel »-, on réfère également à cette unité très importante du rituel de rencontre, par le terme whaikōrero. Sur la centralité de cette unité rituelle dans les rituels de rencontre, voir Salmond (1975). Voir également Gagné (2012: 21).

9. Il avait effectivement lu un texte en français la veille lors de la cérémonie d'inauguration de l'exposition au MCQ. 
rituelle clôt habituellement la partie tapu de la rencontre et permet d'entrer dans sa partie noa (libre de toute restriction religieuse, ordinaire, détendue ; complément et antidote au tapu). La rencontre prit fin après la signature de l'entente de rapatriement au son de waiata (chants) plutôt entraînants et joyeux, lesquels furent applaudis.

La cérémonie qui se tint au MBAM possédait donc les unités rituelles essentielles au déroulement sans risques d'un rituel de rencontre: le karanga, les mihimihi ou whaikörero et le hongi (voir Gagné, 2012 : 12 ; voir également Tauroa et Tauroa, $1986: 141$; Ngata, $2005: 32$ ). Voici, à titre comparatif, des tableaux résumant la structure d'un rituel de rencontre type (fig. 1), celle de la cérémonie au MQB à Paris (fig. 2) et celle de la cérémonie au MBAM à Montréal (fig. 3). On retrouve dans les tableaux des indications quant aux groupes participant à chaque moment ou unité rituelle et au sexe des principaux protagonistes associés à chaque moment ou unité. À Montréal comme à Paris, les manuhiri (invités, soit les Māori) prirent sur eux la responsabilité de toutes les unités rituelles en amont du whaikörero de façon à garantir la bonne marche de la cérémonie puisque tant le retour sûr et sans risque des toi moko vers leurs terres ancestrales que la pérennité de la nouvelle relation en dépendait. Il ressort de façon évidente de ces tableaux que la cérémonie au MBAM était beaucoup plus sobre et moins élaborée que celle de Paris. Entre autres éléments, une des figures qui impressionna l'assistance au MQB n'était pas présente : le kaitātaki, le challenger ou jeune guerrier qui fit un wero

\begin{tabular}{|c|c|c|}
\hline \multirow{8}{*}{ 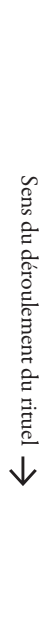 } & Hunga käinga & Manubiri \\
\hline & & Waerea $\widehat{\partial}$ \\
\hline & Wero ${ }^{\pi}$ & \\
\hline & $\begin{array}{l}\text { Karanga } 9 \\
\text { (commence) }\end{array}$ & $\begin{array}{c}\text { Karanga }+9 \\
\text { (répond) }\end{array}$ \\
\hline & Pōwhiri & \\
\hline & Tangi $i$ & $\begin{array}{c}\text { Tang } i \text { ㅇ } \\
\text { (commence) }\end{array}$ \\
\hline & $\begin{array}{l}\text { Whaikörero } \delta^{\lambda} \\
\text { (commence, puis alternance } \\
\text { entre locaux et visiteurs ou tous } \\
\text { les locaux et tous les visiteurs) }\end{array}$ & $\begin{array}{l}\text { Whaikorero } \\
\text { (répond), }\end{array}$ \\
\hline & \multicolumn{2}{|c|}{ Hongi } \\
\hline
\end{tabular}

FIGURE 1. - Structure d'un rituel de rencontre type (tableau réalisé à partir de Salmond, 1975)

ou défi rituel, vêtu d'un piupiu (pagne de lin) et armé d'un taiaha (arme longue en bois sculpté, bâton de combat) qu'il faisait virevolter de façon menaçante. Les Māori présents à Montréal ne portaient pas non plus d'habits traditionnels : ils étaient vêtus de noir et les deux hommes portaient un costume. Signe distinctif, cependant, et signe important de mana, ils arboraient des taonga (trésors, objets ou entités précieux) au cou, c'est-à-dire des pendentifs en jade ou en os.

La plus grande simplicité de la cérémonie correspondait peut-être davantage à la forme que prit la cérémonie de Rouen de mai 2011 au cours

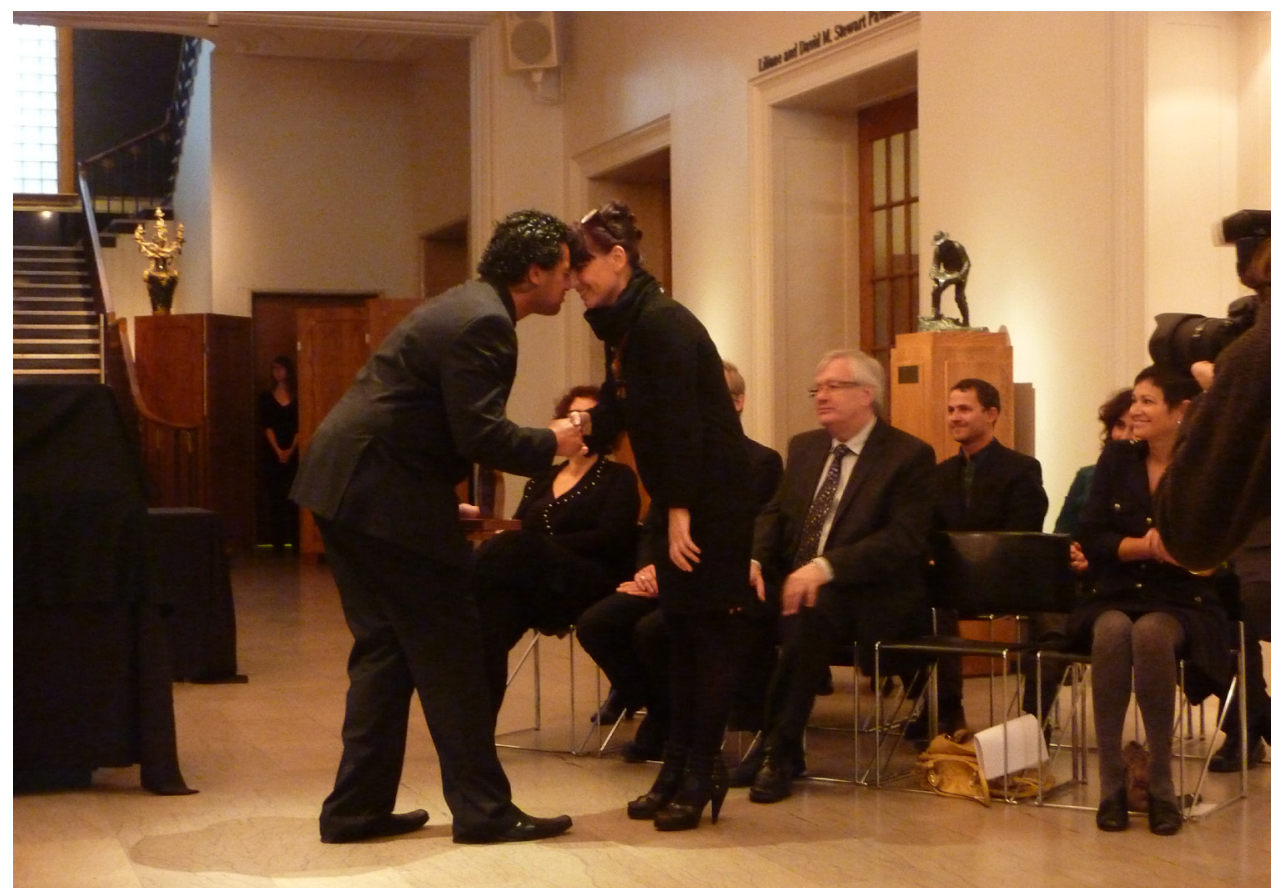

Рното 2. - Hongi accompagnant la remise du koha à Nathalie Bondil (21 novembre 2012, cliché Natacha Gagné) 


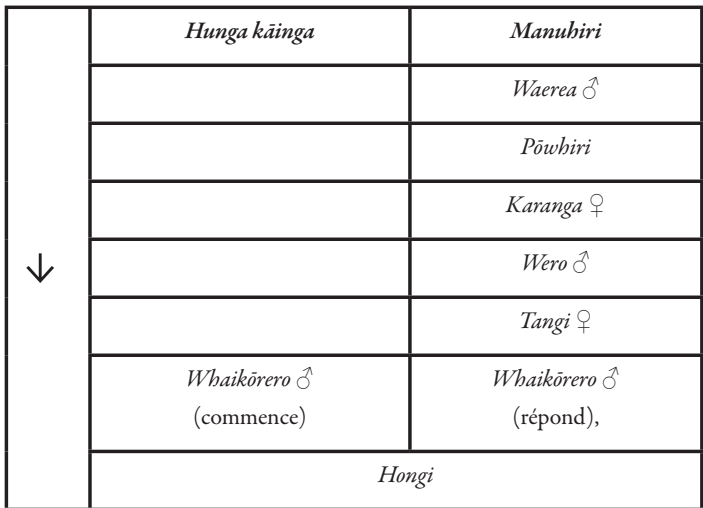

Figure 2. - Structure de la cérémonie de rapatriement au MQB en janvier 2012 (tableau repris de Gagné, $2012: 21$ )

de laquelle le premier toi moko qui faisait partie des collections françaises fut rapatrié, si je me fie aux aperçus que j'ai pu en avoir sur internet (Rostkowski, 2010). Lélaboration du rituel est certainement en partie fonction de l'importance du taonga (trésor) au cour du rituel et de l'entente de rapatriement. Au MQB, en janvier 2012, pas moins de 20 toi moko furent rapatriés, ce qui était, pour le Te Papa, le plus grand nombre de restes ancestraux jamais réunis au sein d'une même entente de rapatriement.

Comme dans le cas de la cérémonie qui se tint au MQB, la disposition des personnes et du toi moko ainsi que la séquence rituelle évoquaient le marae, le lieu traditionnel où se tiennent les rencontres cérémonielles māori (voir Salmond, 1975 et Gagné, 2012, 2013 pour plus de détails). À titre de rappel, le marae consiste en un ensemble clôturé de bâtiments parmi lesquels on retrouve la très caractéristique et très centrale wharenui, la grosse maison ou maison de rassemblement, aussi appelée whare tipuna ou maison ancestrale puisqu elle incarne une des figures ancestrales du groupe. Le marae possède une dimension spirituelle et sacrée et incarne le lien à la terre et aux ancêtres qui y vécurent. Les familles élargies et les tribus possèdent leurs marae. Autrefois, les Māori réservaient le terme marae ou marae atea pour parler de la cour cérémonielle qui se trouvait juste devant la maison (Metge, 1976 : 227). Cet espace est utilisé pour l'accueil des visiteurs et la tenue de rituels de rencontre de diverses natures, dont ceux reliés aux tangihanga ou funérailles. La dépouille du défunt repose alors soit à l'intérieur de la maison, sur le porche ou dans un abri temporaire appelé whare mate (littéralement, maison du mort ou du défunt) installé juste à côté de la wharenui (Salmond, 1975 : 47-48).

Pendant les rituels de rencontre, comme ce fut le cas à l'occasion de la cérémonie au MBAM comme à celle au MQB, les deux groupes impliqués - les

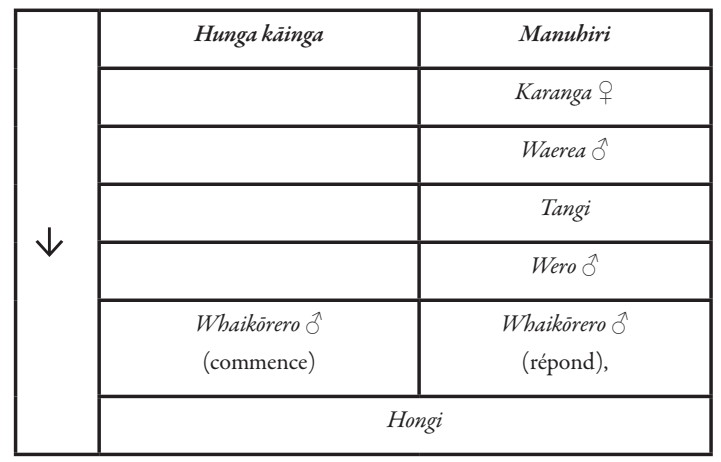

Figure 3. - Structure de la cérémonie de rapatriement au MBAM en novembre 2012

hôtes et les invités - restent clairement séparés en termes spatiaux, se faisant face pendant toute la durée du rituel. Des distinctions en fonction du sexe, de l'âge et du mana (pouvoir spirituel, autorité, statut, prestige) ordonnent également la disposition des gens lors des rencontres rituelles: les hommes et les personnes plus âgées siègent à l'avant. Comme je l'ai expliqué de manière plus détaillée dans mon article de 2012 auquel je renvoie le lecteur, ces distinctions n'impliquent pas des rapports hiérarchiques qui supposeraient que le devant (par rapport à l'arrière), les hommes (par rapport aux femmes), les vieux (par rapport aux plus jeunes) soient supérieurs. Des principes de complémentarité sont plutôt sous-jacents à cette organisation. Ces principes se reflètent également dans les rôles qui sont impartis à chacun durant le rituel (Salmond, 1975, voir également Curchin, 2011 pour une synthèse des débats sur la question). Lors de la cérémonie qui se tint à Montréal, les manuhiri se disposèrent selon ces critères : les hommes devant, disposés par ordre de séniorité et de statut, et les femmes derrière. Sur la première rangée, on retrouvait donc, dans l'ordre, à partir du centre, c'est-à-dire de l'endroit où reposait le toi moko: le kaumatua ou aîné Rahui Papa ; Michel Côté du MCQ devenu rituellement membre du groupe des invités tout comme les autres représentantes du MCQ qui prirent place sur la rangée de derrière avec les femmes; Winton Holmes, le haut-commissaire de la Nouvelle-Zélande à Ottawa; suivis d'autres membres plus jeunes ou moins importants statutairement du groupe parmi lesquels figurait John Scott du Secrétariat de la Convention sur la diversité biologique basé à Montréal qui s'est présenté à moi comme un Aborigène d'Australie. Comme nous l'avons vu plus haut, en plus de Michelle Hippolite, seuls les trois premiers hommes prirent la parole pendant la période des discours cérémoniels (mibimibi).

Tel que déjà mentionné (Gagné, 2012 : 10-11), en Aotearoa-Nouvelle-Zélande, sur le marae, les orateurs sont des hommes ayant atteint le statut 
de kaumatua (aîné). Des critères comme le niveau de connaissance des traditions, de la langue māori ainsi que le mana (statut, autorité, pouvoir spirituel) entrent également en ligne de compte. Dans la plupart des tribus, les femmes ne prennent pas la parole dans cette partie formelle du rituel. Certaines tribus font pourtant exception à cette règle pour les femmes de haut statut (voir Salmond, 1975: 127 ; Tauroa et Tauroa, 1986 : 76-77). Même si la présence du marae était évoquée à Montréal et même si la structure du rituel de rencontre était suivie ainsi que les règles reliées au tapu (aspects sacrés, pollués, polluants, sous restriction) en lien avec le défunt, les marges de liberté étaient plus larges qu'en contexte « traditionnel ", ce qui rendait beaucoup plus facile ou moins problématique le fait que des femmes prennent la parole. Il est toutefois à noter que lors de la cérémonie au MQB, les femmes, dont Michelle Hippolite, ne prirent pas la parole pendant les mihimihi, mais plus tard, après la remise de koha et le hongi effectué par le kaumatua (aîné) Derek Lardelli, qui dirigeait alors la délégation néo-zélandaise, et le ministre français de la Culture, ce qui aurait permis de lever ou de neutraliser le tapu et d'entrer dans la partie noa («libre de toute restriction religieuse, ordinaire, détendue; complément et antidote au tapu») de la rencontre. Sur les marae, une fois que la partie cérémonielle est complétée, les femmes sont libres de prendre la parole comme bon leur semble. Peut-être que le nombre important de toi moko et le tapu les entourant, c'est-à-dire les aspects sacrés, mais également potentiellement dangereux, demandèrent plus de prudence à $\mathrm{Pa}$ ris qu'à Montréal, ce qui pourrait expliquer que les femmes aient attendu la levée du tapu avant de prendre la parole.

Je mentionnais dans une note de mon précédent article (Gagné, 2012 : 10-11) une idée souvent invoquée dans les débats relatifs à l'absence de femmes comme oratrices et au fait qu'elles siègent derrière les hommes lors des rencontres cérémonielles. Celle-ci veut que les femmes, étant celles qui portent la vie et assurent la reproduction, auraient un plus grand mana, et devraient donc être protégées des forces surnaturelles présentes dans les cérémonies sacrées. Cela explique que ce sont les hommes de grand mana et donc plus âgés qui s'exposent davantage. L'âge est donc un facteur à prendre en compte : les femmes qui ne sont plus en âge de procréer peuvent prendre plus de risques. C'est d'ailleurs habituellement une femme âgée ou la plus âgée du groupe qui fait le karanga (appel cérémoniel) et qui marche à l'avant du groupe lors de l'entrée sur un marae étranger. Ce fut le cas lors de la cérémonie au MQB. A Montréal, en l'absence de femme âgée, c'est une femme plus jeune qui exécuta le karanga.

La relative sobriété de la cérémonie de Montréal par rapport à celle de Paris peut également être attribuée, à mon sens, aux contextes nationaux fort différents dans lesquels prenaient place les deux cérémonies. Même si l'événement fut brièvement couvert par les principaux médias du pays, tant francophones qu'anglophones, la cérémonie au MBAM reçut une beaucoup moins grande attention médiatique que celle de Paris. Une des raisons réside, selon moi, dans le statut d'inaliénabilité des collections publiques françaises et dans le fait qu'une loi dut être adoptée pour permettre les rapatriements ${ }^{10}$. Cela fit en sorte de complexifier la procédure et de stimuler un important débat au sein du milieu muséal français. Dans la prochaine section, je donnerai quelques indications permettant de mieux comprendre les contextes québécois et canadien.

\section{Les rapatriements au Québec et au Canada}

À ce jour, le Canada ne s'est pas doté d'une loi qui obligerait les musées à restituer les restes humains et autres objets ethnographiques aux peuples autochtones qui en feraient la demande ${ }^{11}$. Cependant, en 1992, le rapport Tourner la page : forger de nouveaux partenariats entre les musées et les Premières Nations du Groupe de travail sur les musées et les Premières Nations, parrainé conjointement par l'Assemblée des Premières Nations et l'Association des musées canadiens, recommandait entre autres choses de traiter les restes humains avec respect et de prendre en considération les demandes de rapatriements. En lien avec les revendications émanant des Premières Nations, le rapport a mené à une révision des pratiques au sein des musées canadiens.

Le Canada differe donc dans sa démarche des États-Unis qui ont adopté le Native American Graves Protection and Repatriation Act (NAGPRA) en 1990 qui oblige "les institutions muséales subventionnées par le gouvernement américain à rapatrier les ossements humains et les objets qui leur sont associés aux communautés qui en feraient la demande " (Dubuc et Turgeon, 2004 : 10). Il semble pourtant que pareille loi ne soit pas sans problèmes et l'approche canadienne selon laquelle il revient à chaque musée de

10. Pour une synthèse des principaux éléments du contexte français, voir Rostkowski (2010).

11. Voir Fisher (2012) sur la législation fédérale. Précisons que les compétences en matière culturelle et muséale sont partagées entre les provinces et le fédéral. Un nombre limité de musées sont des musées nationaux (voir la liste à http:// www.canada.gc.ca/aboutcanada-ausujetcanada/arts/musgal-fra.html). Les autres musées sont soit privés, semi-privés ou provinciaux. Au Québec, les trois musées provinciaux sont le Musée national des beaux-arts du Québec, le Musée d'art contemporain et le Musée de la civilisation. 
traiter les demandes de rapatriements donnerait de bons résultats :

"Chaque demande est étudiée au cas par cas et l'approche est basée sur la négociation et la conviction. Ce qui est dommage, c'est que ce sont toujours les autochtones qui ont à faire la preuve que leur demande est fondée, même si le milieu commence à mieux comprendre leurs motifs. » (Dubuc, in Baril, 2009)

L'approche n'est donc pas parfaite. Entre autres choses, elle amène une bureaucratisation des demandes. Des conflits de valeurs et de conceptions du monde sont également persistantes (voir, entre autres, Conaty, 2004 ; Noble, 2008 ; Poirier, 2011).

Dans le cas qui nous concerne ici, il est important de savoir que les collections du мBAм ne sont pas "publiques » puisque l'établissement ne l'est pas: d'abord une institution privée, le MBAM est, depuis 1972, une société à but non lucratif de type mixte. Comme tel,

" il reçoit l'aide financière du ministère québécois de la Culture, des Communications et de la Condition féminine pour environ $50 \%$ de son budget de fonctionnement annuel, l'autre moitié étant autogénérée. » ${ }^{12}$

Lors de la cérémonie de rapatriement, parmi les personnes siégeant parmi le groupe des hôtes ${ }^{13}$, il était d'ailleurs intéressant de constater qu'une seule personne ne provenait pas du MBAM. Il s'agissait d'André Lavallée, secrétaire général associé de la région métropolitaine de Montréal, qui représentait le gouvernement du Québec. Celui-ci ne prit d'ailleurs pas la parole pendant la cérémonie.

Certains musées se sont dotés d'une politique en matière de rapatriement dans les suites du rapport de 1992 et d'un débat plus large parmi les autochtones, les professionnels de musée et les chercheurs en sciences sociales (pour un résumé, voir Dubuc et Turgeon 2004). Ce fut le cas notamment du Musée canadien des civilisations (MCC) à Gatineau, au Québec, qui adopta sa politique de rapatriement en mai 2001, laquelle prévoit des critères encadrant les demandes de rapatriements concernant les restes humains, objets de sépulture associés, objets archéologiques et matériel connexe et objets ethnographiques. Ainsi :
«La Société du Musée canadien des civilisations (sMCC) reconnaît le besoin d'envisager, de temps en temps, la possibilité de rapatrier certains objets d'origine autochtone aux peuples autochtones. $»^{14}$

En 2006, le musée aurait d'ailleurs rendu des ossements humains prélevés sur des sites de fouilles archéologiques le long de la rivière $\mathrm{Ou}$ taouais à la communauté de Kitigan Zibi qui fait partie de la nation anishinabeg (algonquine). Élise Dubuc, anthropologue et directrice du programme en muséologie à la Faculté des arts et des sciences de l'Université de Montréal, explique que :

"Le Musée a d'abord soutenu que rien ne permettait de relier ces restes aux Anishinabeg parce que l'Outaouais était une voie de transport et de migration. Mais il a finalement accepté de les leur restituer pour qu'ils les enterrent à leur façon. » (in Baril, 2009)

Les choses ont donc beaucoup changé au cours des dernières années. Quant à savoir si on doit s'inquiéter de l'avenir des musées et de leurs collections, Élise Dubuc ajoute :

«Dans $90 \%$ des cas, on réussit à s'entendre et les autochtones reconnaissent la contribution des musées à la sauvegarde de leur patrimoine. » (in Baril, 2009)

La province de l'Alberta, dans l'ouest du Canada, fait également, depuis quelques années, figure de pionnière en ayant adopté deux lois permettant le retour des objets sacrés ${ }^{15}$ aux groupes issus des Premières Nations de façon à ce qu'ils puissent être utilisés de façon active à des fins cérémonielles. En 2000, l'Alberta adopta donc le First Nations Sacred Ceremonial Objects Repatriation Act (FNSCORA) suivi du First Nations Sacred Ceremonial Objects Amendment Act en 2008 (voir Fisher, 2012). Fisher précise que :

"The FNSCORA was designed to consolidate the role of museums in supporting the needs of First Nations people to practice their traditional values while still preserving heritage." (2012)

Ces lois concernent uniquement le Glenbow Museum (Calgary) et le Royal Alberta Museum (Edmonton).

Ces changements doivent être mis en lien avec le mouvement plus large de décolonisation, no-

12. Voir la section "Historique " du site internet du MBAм à : http://www.mbam.qc.ca/a-propos-du-musee/historique (site consulté le 15 janvier 2013).

13. La délégation du mвAм comprenait des représentants du Conseil d'administration, de l'administration, ainsi que des départements de la conservation, de la restauration, des archives et de la communication. Il est d'ailleurs intéressant de noter qu'aucun représentant amérindien ne figurait parmi les hôtes, ce qui n’était pas le cas la veille lors des cérémonies de bénédiction et d'inauguration au MCQ auxquelles participèrent des représentants des Hurons-Wendat, une nation autochtone de la région de Québec.

14. Cette politique peut être consultée en ligne sur le site du MCC à : http://www.civilisations.ca/files/2011/09/politique-sur-le-rapatriement.pdf (site consulté le 15 janvier 2013).

15. Ces lois portent spécifiquement sur les objets sacrés et ne font pas mention des restes humains. 
tamment dans le domaine de la recherche (voir par exemple Lévesque, 2009) menant à de nombreux partenariats entre chercheurs, établissements non autochtones et communautés autochtones, ainsi quavec le mouvement autochtone à l'international qui a conduit, entre autres, à l'adoption de la Déclaration sur les droits des peuples autochtones par l'Assemblée générale de l'oNu en 2007. À l'article 12, alinéa 1 , on peut lire :

"Les peuples autochtones ont le droit de manifester, de pratiquer, de promouvoir et d'enseigner leurs traditions, coutumes et rites religieux et spirituels; le droit d'entretenir et de protéger leurs sites religieux et culturels et d'y avoir accès en privé ; le droit d'utiliser leurs objets rituels et d'en disposer ; et le droit au rapatriement de leurs restes humains. ${ }^{16}$

Dans un numéro spécial de la revue Anthropologie et sociétés sur la thématique « Musées et Premières Nations ", Dubuc et Turgeon ont identifié les deux tendances générales dans lesquelles s'inscrivent les changements en Amérique du Nord :

1) les nouvelles pratiques entourant les collections autochtones continuent de s'inscrire dans la tradition du musée occidental (européen), mais " on prône la restitution de certains objets sacrés aux communautés autochtones et leur participation accrue dans la préparation d'expositions" (2004 : 11), privilégiant ainsi la négociation et la plurivocalité ;

2) "les Autochtones tendent de plus en plus à prendre leurs distances par rapport aux musées occidentaux et à développer leurs propres pratiques muséales» $(2004: 14)^{17}$.

Ces changements doivent également être mis en lien avec l'évolution des réflexions de nature éthique entourant les restes humains au Québec, au Canada et plus largement dans le monde ${ }^{18}$. Comme le faisait remarquer une journaliste du quotidien Le Devoir dans son reportage sur la cérémonie du 21 novembre 2012 au MBAM, des débats ont eu lieu récemment à propos du statut particulier des restes humains au Québec. J'ai déjà évoqué le cas des ossements rapatriés à la communauté anishnabeg de Kitigan Zibi. Mais la question s'est déjà posée à propos de restes humains non autochtones. Ainsi, «l'exposition récente (2009) des os d'Alexis Lapointe, dit Le Trotteur, à Chicoutimi, ou celle du cadavre du géant Beaupré à l'Université de Montréal, se sont toutes deux soldées par l'enterrement de leurs restes en bonne et due forme " (Paré, 2012) et donc à leur retour à leurs parents ou région d'origine $^{19}$.

La position du conseil d'administration du MBAM quant au toi moko dont il est question dans le présent article me semble tout à fait illustrer les changements en cours. Ainsi :

"Sur la proposition de la direction du mbam, le conseil d'administration a voté à l'unanimité l'an dernier pour cette demande, spécifiant que, selon lui, "il ne s'agit pas réellement d'une restitution, donc d'un transfert de propriété, car la propriété du corps humain est inaliénable”. " (Communiqué de presse, MBAM, 21 novembre 2012) ${ }^{20}$

En entrevue sur les ondes de СвС Radio, $\mathrm{Na}$ thalie Bondil expliquait que lorsqu'on lui demandait si c'était une perte pour le MBAM, elle répondait :

«Not at all, because our mission is really to preserve a spiritual heritage and to respect it and I think what is most important is not to keep a collection like a bank, but really to show that there are different ways to consider culture and it was a perfect example. [...] The circle is closed, because now, we help all those cultures to reactivate their own pride and identity. So, I think also that it's normal to consider those objects not like art objects or curiosity objects, but like something different... relics or human beings. [...] I think that [...] there is not just one interpretation. The New Zealand representatives were very clear on that point. They said that for them, this head was not an art object or an object at all. It was someone, an ancestor. They did not say that their interpretation, their vision, was the only one. The change is that now, we accept to dialogue, we accept to respect other representations on objects. For example, what is good or respectful for the Mãori people could be different for another tribe. What is important is just to have an open mind and to listen to the ways they consider those objects. " ("Cinq à Six ", CBC Radio, 27 novembre 2012)

16. Voir le texte complet de la Déclaration à : http://www2.ohchr.org/french/issues/indigenous/declaration.htm (consulté le 15 janvier 2013).

17. Ces tendances se retrouvent également ailleurs comme en Australie et en Nouvelle-Zélande (Mauzé et Rostkowski, 2007). Sur la Nouvelle-Zélande, voir, par exemple, McCarthy (2011).

18. Pour un résumé sur les débats en France, voir, entre autres, Berger (2008) et Rostkowski (2010).

19. La dépouille d'Édouard Beaupré demeura en possession de l'Université de Montréal de 1907 à 1989. Le corps momifié fut exposé nu derrière une vitrine du laboratoire d'anatomie. À la suite d'une demande de membres de sa famille, "[p] our éviter une autre exposition publique de mauvais goût, l'Université accepte de rendre le corps, mais à la condition qu'il soit incinéré. Les cendres sont envoyées en Saskatchewan où Édouard Beaupré est enfin inhumé, le 7 juillet 1990 » (voir la section réservée à la série « Musée Eden » sur le site de Radio-Canada à : http://musee-eden.radio-canada.ca/montreal-1910/article/2/le-plus-grand-homme-du-pays, site consulté le 15 janvier 2013). Quant aux ossements de celui qui était surnommé "l'homme-cheval », ils furent inhumés en novembre 2009 à Clermont, ville natale d'Alexis Lapointe, dit le Trotteur, après avoir été exposés pendant 35 ans au musée La Pulperie de Chicoutimi (Desmeules, 2009).

20. Dans son allocution, la direction et conservatrice en chef du MBAM, Nathalie Bondil, a également insisté sur cette idée, rappelant la position du CA du MBAM. 


\section{Elle disait encore :}

"C'est pour nous un soulagement de savoir que ces restes humains reposeront désormais sur la terre de leurs ancêtres. C'est aussi justice de penser qu'ils ne seront plus jamais livrés à la curiosité du public ou entreposés comme artefacts dans des réserves muséales. Notre regard s'éduque et nous trouvons aujourd'hui normal que la dignité humaine soit respectée, même si persistent, à mon sens, des questions d'éthique sur ce que nous donnons à voir, notamment dans le cas de certaines expositions de cadavres. Cette problématique demeure très actuelle." (Communiqué de presse, MBAM, 21 novembre 2012)

La vision du MBAM et de sa directrice et conservatrice en chef s'inscrit parfaitement dans ce nouveau modèle d'interaction entre les Premières Nations et les musées qui est en train de s'établir. C'est cet autre modèle que décrivait Gérald Conaty, le conservateur principal d'ethnologie au musée Glenbow de Calgary, un musée qui fut à l'origine d'une controverse ${ }^{21}$ en 1988 qui mena à la création en 1989 du Groupe de travail sur les musées et les Premières Nations au Canada :

"Dans ce nouveau contexte, toutes les parties se considèrent comme égales, même si leurs perspectives different considérablement. Ce nouveau type de relation rend possible la négociation de questions difficiles, lourdes de préoccupations liées à l'identité, au pouvoir (social et politique) et à l'histoire, tout en visant un respect mutuel. » (2004 : 64)

À propos de ce nouveau contexte, Élise Dubuc précise que :

"Les musées ont beaucoup à apprendre de ces échanges parce qu'ils connaissent très peu l'histoire des autochtones et celle des pièces qu'ils possèdent. » (in Baril, 2009)

C'est aussi ce que disait Nathalie Bondil :

"What is important for our publics is really to be open as much as possible towards different interpretations, perspectives, histories... " ("Cinq à Six », СвC Radio, 27 novembre 2012)

\section{Conclusion}

Tout comme la cérémonie s'étant tenue au MQB en janvier 2012 (Gagné, 2012), la cérémonie de novembre de la même année au MBAM a opéré une inversion symbolique en permutant les rapports qui déterminent habituellement le format des rituels de rencontre : alors que ce sont les hunga kāinga (gens du lieu, hôtes) qui déterminent le kawa (protocole) et les tikanga (tradi- tions) qui orienteront le rituel, les Québécois, tout comme les Français avant eux, furent plutôt transportés dans l'univers māori. Cette inversion symbolique est associée à un renversement du rapport politique en faveur des Māori.

La réarticulation des relations que ce renversement suppose s'inscrit dans la longue histoire des luttes māori et de leur projet d'émancipation politique (pour une synthèse, voir Gagné, 2010, 2013). Elle s'inscrit également dans un monde (post) colonial en train de se définir. Au Québec et au Canada, un processus lent de changement répondant favorablement aux demandes autochtones est amorcé depuis quelques années. Ce processus s'apparente à celui qui est également en cours en Nouvelle-Zélande, pays qui partage une partie de son histoire coloniale avec le Canada. Ce vent de changement touche notamment les institutions muséales, comme nous l'avons montré.

Bien que présentant de grandes similarités au plan structurel, les cérémonies de rapatriement de toi moko ayant eu lieu en 2012 à Paris et à Montréal n'étaient pourtant pas tout à fait les mêmes. D'autres facteurs que ceux énumérés plus haut ont certainement pu jouer dans certains choix faits par les participants à ces cérémonies. Par exemple, certains membres de la délégation māori ont mentionné avoir voulu faire quelque chose de plus simple qu'à Paris et de limiter leur exposition aux médias. La personne du kaumatua (â̂né), qui n'était pas non plus la même, peut également avoir eu un impact sur l'envergure, le format et le contenu du rituel de rencontre : le leader donne habituellement sa couleur - et celle de sa tribu - à la cérémonie. Cependant, comme j'ai tenté de le montrer ici, les différences seraient à comprendre dans les contextes (post) coloniaux qui furent ceux des deux cérémonies analysées. Ces contextes gagnent donc à être appréhendés à l'aune de l'analyse minutieuse des actions des sujets.

\section{BIBLIOGRAPHIE}

BARIL Daniel, 2009 (01/11). Un totem mortuaire est-il un simple objet ou l'enveloppe de restes humains ?, Journal Forum (http://www. nouvelles.umontreal.ca/recherche/scienceshumaines-lettres/un-totem-mortuaire-est-ilun-simple-objet-ou-lenveloppe-de-restes-humains.html).

Berger Laurent, 2008 (26/09). Des restes humains, trop humains?, La Vie des idées (http://www.laviedesidees.fr/Des-restes-humains-trop-humains.html). 
Boulay Roger, 2012. L'initiative du Muséum d'histoire naturelle de Rouen : le contexte local, Journal de la Société des Océanistes 134, pp. 25-27.

Conaty Gerald T., 2004. Le rapatriement du matériel sacré des Pieds-Noirs : deux approches, Anthropologie et Sociétés 28 (2), pp. 63-81.

Curchin Katherine, 2011. Pākehā Women and Māori Protocol: The Politics of Criticising Other Cultures, Australian Journal of Political Science 46 (3), pp. 375-388.

Desmeules Sylvain, 2009 (07/11). Un dernier repos pour Alexis le Trotteur, Le Soleil (http://www.lapresse.ca/le-soleil/actualites/ les-regions/200911/07/01-919514-undernier-repos-pour-alexis-le-trotteur. php?utm_categorieinterne $=$ trafficdrivers $\& u$ tm_contenuinterne=cyberpresse_vous_suggere_4305117_article_POS1).

Dubuc Élise et Laurier Turgeon, 2004. Musées et premières nations : la trace du passé, l'empreinte du futur, Anthropologie et Sociétés 28 (2), pp. 7-18.

Fisher Darlene, 2012. Repatriation Issues in First Nations Heritage Collections, Journal of Integrated Studies 1 (3) (http://jis. athabascau. $\mathrm{ca} /$ index.php/jis/article/view/79).

GAGnÉ Natacha, 2010. Les espaces multiples de l'affirmation de l'autonomie maaori, in N. Gagné et M. Salaün (éds), Visages de la souveraineté en Océanie, Paris, L'Harmattan, Cahiers du Pacifique Sud contemporain 6, pp. 41-67.

—, 2012. Affirmation et décolonisation : la cérémonie de rapatriement par la France des toi moko à la Nouvelle-Zélande en perspective, Journal de la Société des Océanistes 134, pp. 5-24.

-, 2013. Being Mãori in the City: Indigenous Everyday Life in Auckland, Toronto, University of Toronto Press.

Harrison Julia D. et Bruce Trigger, 1988. 'The Spirit Sings' and the Future of Anthropology, Anthropology Today 4 (6), pp. 6-10.

LÉVESQUE Carole, 2009. La recherche québécoise relative aux peuples autochtones à l'heure de la société du savoir et de la mobilisation des connaissances, in Natacha Gagné, Thibault Martin et Marie Salaün (éds), Autochtonies: vues de France et du Québec, Québec, Presses de l'Université Laval, pp. 455470.
MauzÉ Marie et Joëlle Rostkowski, 2007. La fin des musées d'ethnographie? Peuples autochtones et nouvelles perspectives muséales, Le Débat 5 (147), pp. 80-90.

McCarthy Conal, 2011. Museums and Mãori: Heritage Professionals, Indigenous Collections, Current Practice, Walnut Creek, Left Coast Press.

Metge Joan, 1976. The Maoris of New Zealand Rautahi, London, Routledge and Kegan Paul.

Ngata Paratene, 2005. Death, Dying and Grief, in Margot Schwass (ed.), Last Words: Approaches to Death in New Zealand's Cultures and Faiths, Wellington, Bridget Williams Books et The Funeral Directors Association of New Zealand, pp. 29-40.

Noble Brian, 2008. Owning as Belonging/ Owning as Property: The Crisis of Power and Respect in First Nations Heritage Transactions with Canada, in Catherine Bell and Val Napoleon (eds), First Nations Cultural Heritage and Law: Case Studies, Voices, Perspectives, Vancouver, UBC Press, pp. 465-488.

ParÉ Isabelle, 2012 (22/11). Le MBAm retourne une tête maorie en terres ancestrales, $L e D e-$ $\operatorname{voir}$ (http://www.ledevoir.com/culture/actualites-culturelles/364555/le-mbam-retourneune-tete-maorie-en-terres-ancestrales).

Peltier Philippe et Magali Mélandri, 2012. Chronologie concernant les têtes tatouées et momifiées māori ou toi moko (aussi connues sous le terme de moko mokai), Journal de la Société des Océanistes 134, pp. 28-30.

PoIrIer Claire, 2011. Drawing Lines in the Museum: Plains Cree Ontology as Political Practice, Anthropologica 53 (2), pp. 291-303.

Rosenblatt Daniel, 2011. Indigenizing the City and the Future of Maori Culture: The Construction of Community in Auckland as Representation, Experience, and Self-Making, American Ethnologist 38 (3), pp. 411429.

Rostкоwsкi Joëlle, 2010. Le retour de quelques têtes maories et autres actualités, Recherches amérindiennes au Québec XL (3), pp. 103109.

Salmond Anne, 1975. Hui: A Study of Maori Ceremonial Gatherings, Auckland, Reed Books.

Tauroa Hiwi et Pat Tauroa, 1986. Te Marae: A Guide to Customs \& Protocol, Auckland, Reed Books. 